\title{
A Note on Spelling
}

I have followed the system established by the Library of Congress for transliteration of Russian words and names. I have made exceptions, however, for well-known persons and places whose names are more familiar to readers in other spellings: for example, Gorky, Kazan, Lunacharsky, Mayakovsky, Meyerhold, Mikoyan, Pokrovsky, Rimsky-Korsakov, Stanislavsky, Tchaikovsky. 
